\title{
LUCHANDO CONTRA UNA DE LAS CAUSAS DE INVALIDEZ: ANTECEDENTES, CONTEXTO SANITARIO, GESTACIÓN Y APLICACIÓN DEL DECRETO DE VACUNACIÓN OBLIGATORIA CONTRA LA VIRUELA DE 1903*
}

\author{
$M^{a}$ Isabel Porras Gallo \\ Unidad de Historia de la Medicina. \\ Facultad de Medicina-Centro Regional de Investigaciones Biomédicas (CRIB). UCLM.
}

\section{RESUMEN}

En el presente trabajo se examinan los antecedentes legislativos del Decreto de 1903, el contexto sanitario en el que se gestó, su proceso de gestación y aprobación, y se ofrece una aproximación a su aplicación posterior. Con ello se muestra cómo los factores político-económico-sociales, legislativos, profesionales y sanitarios condicionaron la lenta generalización de la vacunación antivariólica en España en los siglos XIX y XX.

PALABRAS CLAVE: vacunación antivariólica, historia de la viruela, historia de la salud pública, España, siglos XIX y XX.

\section{SUMMARY}

In this paper we analyse the legislative precedents of the 1903 Decree, the sanitary conditions in which it had been developing, his gestation process and his passing, and we show an approach to its subsequent implementation. We show how the political-economic-social, legislative, professional and sanitary factors would be determine the slowly generalization of the smallpox vaccination in Spain during the $19^{\text {th }}$ and $20^{\text {th }}$ centuries.

KEY WORDS: smallpox vaccination, history of smallpox, history of public health, Spain, $19^{\text {th }}$ and $20^{\text {th }}$ centuries.

* Este trabajo se ha realizado en el marco del proyecto del Ministerio de Ciencia y Tecnología ( ${ }^{\circ}$ de referencia: BHA2001-2979-C05-05) y del de la Consejería de Sanidad de la JCCM ( ${ }^{\circ}$ de Expediente: 01043$)$. 


\section{INTRODUCCIÓN}

La celebración del centenario del Real Decreto de 15 de enero de 1903 de vacunación obligatoria contra la viruela en España constituye una buena oportunidad para ocuparse de una enfermedad que durante siglos fue una de las principales causas de muerte y una de las posibles razones de invalidez para la población que sobrevivía a dicho proceso morboso ${ }^{1}$. De hecho, este carácter invalidante motivó manifestaciones como las de Avervek, médicocirujano de Bremen, quien hace algo más de un siglo llamó la atención sobre el hecho de que aquellos que no sucumbían a la enfermedad, «maldecían mil veces la vida», ya que ninguno se libraba «de quedar desfigurado» ${ }^{2}$. Precisamente, apelando a esta condición invalidante de la viruela, un médico de Huesca defendió y justificó la obligatoriedad de la vacunación y revacunación contra la viruela en el IX Congreso Internacional de Higiene y Demografia de 1898. En su opinión, con la adopción y aplicación de dicha medida se lograría que desaparecieran

«ciertos peligros que, si no ocasionan la muerte del individuo inmediatamente después de haber sufrido la invasión variolosa, en cambio le dejan tachas indelebles en el ejercicio de sus funciones de nutrición y hasta en las de relación, que constituyen doble desgracia, a los seres que tienen la mala ventura de verse sumidos en tales circunstancias» ${ }^{3}$.

A la vista de las palabras que acabo de reproducir, parece bien apropiado el abordaje de la viruela en el año europeo de las discapacidades.

Los objetivos principales que, en este doble marco, pretendo cubrir con el presente trabajo son los siguientes. Mostrar primeramente cuáles fueron los antecedentes legislativos del citado Real Decreto, dar cuenta seguidamente del contexto sanitario en el que dicho Decreto se gestó, así como del proceso mismo de su gestación y aprobación; y, por último, exponer sus rasgos más

1 Para obtener una visión sintética acerca de lo que ha sido la historia de la viruela y la lucha contra dicha enfermedad, resulta muy útil el trabajo de CROSBY, A.W. (1993), «Smallpox». En: KIPLE, K.F. (ed.), The Cambridge World History of Diseases, Cambridge, CUP, 1008-1013.

2 AVERVEK (1881), Sobre la vacunación y la obligación de vacunarse. Estudio científico popular del Dr., trad. esp. por Juan Cruz y Vázquez, Madrid, Imprenta de Enrique Teodoro, p. 15.

3 Laín Sorrosal (1900), Vacunación obligatoria. En: SAlcedo Ginestal, E. (dir.) Actas y Memorias del IX Congreso Internacional de Higiene y Demografía celebrado en Madrid en los dias 10 al 17 de abril de 1898..., t. II, Madrid, Imprenta de Ricardo Rojas, 57-59, p. 59. (La cursiva es mía). 
destacados y ofrecer una imagen siquiera somera de su aplicación posterior. A través de ello se irán poniendo de relieve los principales factores responsables de la lenta generalización de la vacunación antivariólica en España tras su rápida difusión inicial. A tal objeto, he consultado monografías sobre la viruela, Actas y Memorias de los Congresos Internacionales de Higiene, las principales revistas científico-profesionales y algunas obras generales de carácter demográfico-sanitario del último tercio del siglo XIX y primeras décadas del $\mathrm{XX}^{4}$. He utilizado los datos estadísticos incluidos en algunas de las publicaciones citadas, si bien para el siglo XX me he servido de los proporcionados en el Centro Nacional de Epidemiología $a^{5}$. Puntualmente, he recurrido a la prensa obrera.

\section{LA PRÁCTICA DE LA VACUNACIÓN ANTIVARIÓLICA EN LA PRIMERA MITAD DEL SIGLO XIX}

Como es bien sabido, la implantación de la variolización -primera medida profiláctica útil contra la viruela- no estuvo exenta de importantes dificultades y oposiciones en los países de nuestro entorno. Algo similar ocurrió con la vacuna puesta a punto por Edward Jenner en $1795^{6}$, como señaló el médico catalán Francisco Piguillem 7 , al dar cuenta de la primera vacunación en nuestro país llevada a cabo por él mismo en diciembre de $1800^{8}$. Ahora bien, estas dudas y reservas frente al nuevo recurso protector contra la viruela no impi-

4 Agradezco a Ignacio Díaz-Delgado, bibliotecario de la RANM su inestimable ayuda para localizar algunas de las fuentes.

5 De hecho, me he servido de la información que, amablemente y con toda generosidad, me ha facilitado Ferrán Martínez Navarro, Jefe de Área de Vigilancia de Salud Pública de dicho centro (ISCIII).

6 Este autor publicó sus primeros resultados en 1798, bajo el título An Inquiry into the Causes and Effects of Variolae Vaccinae, a Disease, Discovered in some of the Western Counties of England, particularly Gloucestershire, and known by the Name of Cow Pox, London.

7 Entre los trabajos dedicados a este médico, cabe señalar la reciente e interesante aportación de DANON, J. (2000), «Estudi preliminar sobre Francesc Piguillem en la Medicina catalana del seu temps». En: Piguillem, F. (1801), La vacuna en España o cartas familiares sobre esta inoculación escritas a la señora **, Puigcerdá, Fundación Uriach, pp. 5-31.

8 Piguillem, F. (1801), La vacuna en España o cartas familiares sobre esta inoculación escritas a la señora **, Barcelona, Sierra y Oliver Martí, p. 16. He manejado la edición fácsimil publicada en 2000 por la Fundación Uriach. 
dieron que su práctica se difundiera rápidamente por Europa ${ }^{9}$, incluyendo dentro de nuestras fronteras ${ }^{10}$. De hecho, la difusión de la vacunación se produjo con celeridad desde 1799 hasta 1803, año de la famosa expedición Balmis organizada por España para propagar la vacuna en los territorios de ultramar ${ }^{11}$, y momento en el que se inició la regresión de esta práctica, tanto en otros países europeos como, sobre todo, en el nuestro ${ }^{12}$.

Entre las razones que han sido apuntadas para justificar dicha regresión en la práctica de la vacunación en nuestro país, figura la carencia de un marco

9 Sobre este tema, puede verse: DARMON, P. (1986), La longue traque de la variole, Paris, Perrin, pp. 175-198. Una interesante revisión sobre la recepción y aplicación práctica de la variolización y la vacunación contra la viruela en Europa y Oriente, contextualizada en el marco amplio de las vacunaciones, son algunos de los trabajos reunidos en la obra de MoULIN, A.-M. (dir) (1996), L'aventure de la vaccination, Paris, Fayard, pp. 1-121.

10 Entre las aportaciones que se ocupan de la inoculación y la introducción de la vacuna en nuestro país, figuran las siguientes: RUMEU DE ARMAS, A. (1940), «La inoculación y la vacunación antivariólica en España (Datos para la historia de la medicina española en los siglos XVIII y XIX)», Medicina Española, tomo IV, 19, 46-59; 20, 143-159; 21, 233-241, 22, 317-329; 392-410; RIERA, J. (1985), «Los comienzos de la inoculación de la viruela en la España Ilustrada», Medicina e Historia, 1984 (8), 4-26; DEMERSON, P. de (1993), «La práctica de la variolización en España», Asclepio, 45-2, 3-39; OlaGÜE DE Ros, G.; Astrain GALlarT, M. (1994), «Una carta inédita de Ignacio Ruiz de Luzuriaga (1763-1822) sobre la difusión de la vacuna en España (1801)», Dynamis, 14, 305-337; OlaGÜE DE Ros, G.; ASTRAIN GALlaRT, M. (1994), «Propaganda y filantropismo: Los primeros textos sobre la vacuna Jenneriana en España», Medicina e Historia, 56, I-XVI; OlAGÜE DE ROS, G. (1995), «La introducción de la vacunación jenneriana en España (1799-1805)». En: BARONA, J. L. (ed.), Malaltia i Cultura, València, Seminari d'Estudis sobre la Ciencia, 251-273.

11 Información acerca de esta expedición, figura en: DíAZ DE IRAOLA, G. (1948), La vuelta al mundo de la expedición de la vacuna, Sevilla, CSIC; BALLESTER AÑóN, R. (1986), «Repercusiones y significado de la Real Expedición Filantrópica de la vacuna». En: La Ilustración Española. Actas del Coloquio Internacional celebrado en Alicante, 1-4 octubre de 1985, Alicante, 35-49; BAlaguer, E. (1987), «Estudio introductorio». En: SARTHE, M. de la, Tratado histórico-práctico de la vacuna, Valencia, (ed. fácsimil de la original traducida por Balmis y publicada en 1803); FRIAS NuÑEZ, M. (1992), Enfermedad y sociedad en la crisis colonial del Antiguo Régimen. (Nueva Granada en el tránsito del siglo XVIII al XIX: Las epidemias de viruela), Madrid, CSIC, pp. 185-223; RAMIREZ MARTín, S. (1998), La Real Expedición filantrópica de la vacuna en la Real Audiencia de Quito, Tesis Doctoral. Facultad de Geografía e Historia. UCM. Sobre esta expedición, véase igualmente el trabajo de esta misma autora que figura en el presente volumen.

12 OlagüE DE Ros, G. (1995), pp. 251 y 260. Sobre este mismo tema, además de los trabajos citados en la nota anterior, véase también la aportación realizada en este volumen por G. Olagüe y M. Astrain. 
legislativo e institucional que permitiera realmente salvaguardar la práctica vacunal $^{13}$. De hecho, la Real Cédula de 21 de abril de 1805, por la que debía establecerse una sala de vacunación en los hospitales de las capitales de España, fue la primera normativa legal ${ }^{14}$. Como se puede ver, se dio tardíamente y fue consecuencia de la expedición de Balmis. Pues bien, los años que siguieron del diecinueve tampoco aportaron mucho más en materia legislativa. Así, la Real Orden de 14 de agosto de 1815 recordaba, a petición de la Academia de Medicina, el cumplimiento de la Real Cédula de 1805. El mismo fin tenía la Circular del Consejo Real de 8 de julio de 1817 para con la R.O. de 1815. Por su parte, el Reglamento de 2 de agosto de 1848, señalaba la obligación de declarar las enfermedades epidémicas y examinar el estado «en que se encuentra la propagación de la vacuna, procurando fomentarla, y dando cuenta cada año del estado de sus investigaciones, con las observaciones que consideren convenientes» ${ }^{15}$. Las palabras reproducidas reflejan unas obligaciones bastante vagas sin acompañamiento de medida alguna tendente al establecimiento de un marco institucional que hiciera realmente viable la práctica vacunal de modo permanente y su extensión progresiva a capas cada vez mayores de población. Este mismo talante se mantuvo en otras disposiciones legislativas posteriores ${ }^{16}$. Algunas de ellas tuvieron finalidades muy concretas, como la R. O. de 15 de abril de 1858, por la que el Gobierno aprobaba un envío de vacuna fresca y de buena naturaleza a las Islas Filipinas, al objeto de poder luchar efectivamente contra las epidemias variolosas allí desarrolladas ${ }^{17}$; o la R.O. de 27 de diciembre de 1860, en la que se dispuso, en vista del informe de la Academia de Medicina en Madrid, que en las vacunaciones y revacunaciones se tenía que hacer uso de virus de buena calidad ${ }^{18}$.

Como vemos, durante algo más de cincuenta años se legisló poco y su finalidad fue en la mayoría de las ocasiones actuar como recordatorio de la necesidad de cumplimiento de esa primera medida, recogida en la Real Cédu-

13 OlagüE DE Ros, G. (1995), p. 252.

14 Dirección General DE SANIDAD (1903), Datos históricos acerca de la vacuna en España. Leyes y Decretos contra la viruela, Madrid, pp. 55. De esta medida se ha ocupado: SANTAMARÍA, E. (1990), «Las salas de vacunación en los hospitales peninsulares a principios del siglo XIX», Dynamis, 10, 303-311.

15 Dirección General De SANIDAD (1903), pp. 72-73.

16 Aunque se ha efectuado una revisión de las disposiciones legislativas relacionadas con la viruela y la práctica vacunal, el lector debe tener presente que una revisión exhaustiva de las mismas se escapa de los objetivos de este trabajo.

17 DiRECCIÓN GENERAL DE SANIDAD (1903), pp. 73-77.

18 Dirección GENERAL DE SANIDAD (1903), p. 77. 
la de 1805. En otras, se buscó atender a algunos de los problemas concretos presentados con la práctica de la vacunación, especialmente durante las epidemias de viruela. De ahí la necesidad de recordar que se usara para ello linfa de buena calidad.

Por otro lado, a diferencia de lo que ocurrió en la mayoría de los países europeos ${ }^{19}$, el nuestro «no contó con instituciones, públicas o privadas, dedicadas exclusivamente a velar por la expansión de la vacuna» ${ }^{20}$.

Otro factor negativo que se sumaba a ese marco legislativo e institucional escaso, era la ausencia de una potente infraestructura burocrática. Recordemos que la defensa de la salud pública española durante los siglos XVIII y XIX estuvo articulada sobre las Juntas de Sanidad, de las que dependía en exclusiva la prevención contra la extensión de las enfermedades epidémicas ${ }^{21}$. Esta organización fue recogida en la Ley de Sanidad de $1855^{22}$, que extendió al ámbito sanitario el modelo centralista napoleónico imperante ya en la Administración pública ${ }^{23}$. Ahora bien, con la Ley de 1855 se sustituyó el sistema cuarentenario por otro basado en la inspección médica realizada por «delegados facultativos», nombrados por el Gobierno, para visitar lugares de inminentes epidemias o contagios, quedando la vigilancia en tiempo de calma epidémica en manos de los Subdelegados de Medicina y Cirugía ${ }^{24}$. Sin embargo, como denunció años más tarde Pulido, los Subdelegados no ejercían en la práctica dicha labor de inspección sanitaria, labor que por otra parte tampoco era remunerada ${ }^{25}$. Como vemos, nuestro sistema, que se mantuvo prácticamente sin cambios hasta la Instrucción General de Sanidad (1904) dejaba mucho que desear, y debe entenderse como una muestra de nuestro retraso en

19 Información acerca de lo ocurrido en otros países de nuestro entorno, figura en: OLAGÜE DE Ros, G. (1995), pp. 261-262.

20 Olagüe De Ros, G. (1995), p. 261.

21 Rodríguez OCAÑA, E. (1994), De la Junta de Sanidad al Instituto de Higiene. En: Historia y Medicina en España. Homenaje a Luis S. Granjel, Valladolid, Junta de Castilla y León, 237-249, p. 237.

22 Cfr. Rodríguez Ocaña, E. (1994), p. 237.

23 Cfr. Beltrán Villalva, M. (1995), La Administración. En: Carr, R. (coord.), La época de Franco (1939-1975). Política, Ejército, Iglesia, Economía y administración, Madrid, Espasa Calpe, pp. 557-637.

24 Molero Mesa, J.; Jiménez Lucena, I. (2000), Salud y burocracia en España. Los Cuerpos de Sanidad Nacional (1855-1951), Revista Española de Salud Pública, 74 (monográfico), 45-79, p. 50.

25 Pulido Fernández, Á. (1902), Sanidad Pública en España y ministerio social de las clases médicas, Madrid, Tip. E. Teodoro, pp. 64-66, 68. 
materia sanitaria, en consonancia con la situación general de nuestro país en el siglo XIX marcada por la inestabilidad política y una no muy boyante situación económica, como consecuencia de una débil Revolución Industrial y de la existencia de una débil burguesía.

Pues bien, a todo lo anterior habría que añadir otro factor que parece que pudo condicionar de forma importante la trayectoria que, en nuestro país, adoptó el tema de la vacunación contra la viruela. Me refiero a la profunda crisis, que -tanto a nivel educativo como en lo referente al ejercicio-, estaba sufriendo la profesión médica y al complejo proceso de reorganización en el que estuvo inmersa desde los últimos años del siglo XVIII ${ }^{26}$. Esta situación probablemente explique que dicha profesión no realizara inicialmente las necesarias iniciativas para lograr el monopolio de la práctica de la vacunación, disputándose dicha práctica cirujanos y médicos, junto a barberos, sangradores y charlatanes ${ }^{27}$.

La suma de todos los factores enunciados propició que el grado de cumplimento de la práctica de la vacunación y de la revacunación contra la viruela no fuera demasiado alto. A ello contribuyó también el miedo de la población hacia dicha medida, que recelaba de su efectividad y temía la transmisión de otras enfermedades conjuntamente con la linfa vacunal. Aunque desde la Medicina se relacionaba este estado de ánimo y actitud de la población con su incultura, conviene recordar que a él habían contribuido no sólo las autoridades políticas, sino también los propios médicos. De hecho, esas ideas fueron vertidas por los antivacunistas para apoyar sus posturas, pero también figuraron en algunos informes médicos como el dictamen del Consejo de Sanidad en respuesta a la comunicación mandada por el Ministro de la Gobernación con motivo de la epidemia variolosa registrada en las Islas Filipinas en 1855. En él, se aceptaba la «degeneración de la vacuna» y se explicaba por no ser el cow-pox una «enfermedad humana» ni una "semilla humana» ${ }^{28}$. Consecuencia del escaso cumplimiento y difusión que la práctica de la vacunación tuvo en nuestro país fue que la viruela, una de las «enfermedades evitables», siguió constituyendo un importante problema sanitario en España, siendo endémica en muchas locali-

26 Peset, M.; PeSET, J.L. (1974), La universidad española, Madrid, Taurus; AlbARRACín TEulón, A. (1974), «La asistencia médica en la España rural durante el siglo XIX», Cuadernos de Historia de la Medicina española, 13, 133-204.

27 OlagüE de Ros, G. (1995), p. 252. Sin embargo, como ha indicado este mismo autor, la Academia de Medicina solicitó en 1801 autorización para dedicar en su seno una sala para vacunaciones gratuitas y, en 1803, elaboró y presentó un plan mucho más ambicioso, véanse: pp. 264-265.

28 DireCción GeNERAL DE SANIDAD (1903), 73-77, p. 74. 
dades y, como se pondrá de relieve, dando lugar a frecuentes epidemias no sólo a lo largo del siglo XIX sino también en el $\mathrm{XX}^{29}$.

\section{SITUACIÓN DE LA VACUNACIÓN ANTIVARIÓLICA DURANTE EL SEXENIO REVOLU- CIONARIO Y PRIMEROS AÑOS DE LA RESTAURACIÓN}

Precisamente, en un ambiente bastante diferente, el del sexenio revolucionario y primeros años de La Restauración, y con unos profesionales sanitarios algo mejor instalados en la sociedad, la presión de estas epidemias contribuyó a mantener periódicamente un debate sobre la viruela y, con ello, se fueron propiciando ciertos cambios que posibilitaron algunas transformaciones que entrañaron un cierto efecto positivo en lo que al cumplimiento de la práctica de la vacunación se refiere. En este sentido, es preciso destacar, por un lado, las sesiones que la Real Academia de Medicina dedicó a ocuparse de «la terapéutica y la profilaxis de la viruela» ${ }^{30}$; $y$, por otro, el dictado de algunas medidas legislativas que tenían como finalidad el establecimiento de un cierto marco institucional — público y privado - que pudiera permitir la propagación y aplicación de la práctica de la vacunación. De hecho, por R.O. de 24 de julio de 1871 se creó un Instituto Nacional de Vacuna, bajo la dirección de Fomento y dependiente de la Academia de Medicina ${ }^{31}$, que tendría un escaso funcionamiento ${ }^{32}$. Este hecho, como ha señalado Ricardo Campos y muestra también en este mismo volumen, no estuvo exento de problemas, provocando enfrentamientos con la Comisión Central de Vacunación del Instituto Médico

29 PÉReZ MoRedA, V. (1980), Las crisis de mortalidad en la España interior (siglos XVIXIX), Madrid, Siglo XXI, pp. 419-422; LóPEZ PIÑERO, J. Ma (1964), El saber médico en la sociedad española del siglo XIX. En: LóPEZ PIÑERO, J. Ma; GARCíA BALleSTER, L.; FAUS SEVILla, P., Medicina y sociedad en la España del siglo XIX, Madrid, Sociedad de Estudios y Publicaciones, pp. 31-108, p. 106; MAtilla, V. (1984), Historia de la Real Academia Nacional de Medicina, Madrid, 1984, p. 99.

30 DisCURSO acerca de la preservación de las viruelas, leído a la Real Academia de Medicina de Madrid, por su socio numerario, Dr. D. Francisco Méndez Álvaro, El Siglo Médico, [921, 20-8-1871, 533-535; 923, 3-9-1871, 563-566; 925, 17-9-1871, 599-601; 926, 24-9-1871, 612-614; 928, 8-10-1871, 644-648; 930, 22-10-1871, 680-684; 938, 17-12-1871, 806-809].

31 DiRECCIÓN GENERAL DE SANIDAD (1903), p. 78.

32 CAMPos Marín, R. (2001), «La vacunación antivariólica en Madrid en el último tercio del siglo XIX. Entre el espacialismo médico y el mercantilismo», Medicina e Historia, 4 (2001), cuarta época, 1-15, pp. 3-4. Sobre este tema, véase también el trabajo de este autor en este mismo volumen. 
Valenciano ${ }^{33}$ y con la Real Academia de Medicina que aspiraba también a fundar su propio Centro de Vacunación ${ }^{34}$. En efecto, así fue expresado por Méndez Álvaro en su discurso del 20 de mayo de 1871, colofón del citado debate que, sobre la «terapéutica y profilaxis de las viruelas», se había celebrado en la Academia de Medicina ${ }^{35}$. Además del Centro de Vacunación, juzgaba Méndez Álvaro necesario «el ordenamiento de un buen sistema de vacunación, extendido a todos los pueblos» ${ }^{36}$, reclamaba que la vacunación fuera realizada «por hombres de conocimientos especiales», y remarcaba la ayuda que «las sociedades médicas» podían prestar tanto en la preservación de las viruelas como en las tareas de divulgación sanitaria a fin de vencer las resistencias de la población frente a la vacunación, «desvaneciendo los errores y preocupaciones que entre el vulgo suelen acreditarse» e inculcando «la utilidad de la revacunación $»^{37}$.

Algo más de un año después de la creación del Instituto Nacional de Vacuna, por R.O. de 14 de diciembre de 1872 se determinó que, por ser «industria libre y lícita para los Profesores de la ciencia de curar» ${ }^{38}$, no era necesaria la autorización del Gobierno para que los médicos establecieran Institutos de vacunación. Con esta medida, quizás motivada, por un lado, por las presiones de ciertos sectores de la clase médica, y, por otro, por el convencimiento de la imposibilidad del Estado de asumir adecuadamente las tareas relativas a la práctica de la vacunación -recordemos las dificultades y el escaso funcionamiento del Instituto Nacional de Vacuna-, se consagró el libre mercado de la vacuna. Ello permitió una cierta proliferación de centros privados de vacunación en los años siguientes.

Como se ha visto, según Méndez Álvaro, otro de los elementos necesarios para poder llevar a cabo la práctica vacunal era disponer de un personal médi-

33 CAMPos Marín, R. (2001), pp. 3-4.

34 DisCURSO acerca de la preservación de las viruelas, leído a la Real Academia de Medicina de Madrid, por su socio numerario, Dr. D. Francisco Méndez Álvaro, El Siglo Médico, 938, 17-12-1871, 806-809. Véanse sobre todo pp. 807-809.

35 Ibidem.

36 Ibidem. Con el fin de extender realmente la vacunación a todos los pueblos, José Guillén, médico titular de Arganda, proponía al ministro de Fomento «la creación de médicos higienistas». EXPOSICIÓN AL EXCMO. SR. MinisTRO DE FOMENTO (por el Licenciado José Guillén), El Siglo Médico, 925, 17-9-1871, pp. 603-605.

37 DisCURSO acerca de la preservación de las viruelas, leído a la Real Academia de Medicina de Madrid, por su socio numerario, Dr. D. Francisco Méndez Álvaro, El Siglo Médico, 938, 17-12-1871, 806-809, véanse sobre todo pp. 807-809.

38 DiRECCIÓN GENERAL DE SANIDAD (1903), p. 78. 
co apropiado para acometer semejante tarea. Dar respuesta a esta necesidad no parecía sencillo, recordemos el escaso nivel de modernización y la escasa atención que se prestaba en esos momentos a la denominada Sanidad Civil, especialmente a la Sanidad terrestre ${ }^{39}$. De hecho, no sería hasta la reorganización de 1892, bajo la amenaza de la epidemia de cólera que se estaba desarrollando en Europa (Hamburgo, Bélgica, Francia...), cuando se produjo la creación de los Inspectores Provinciales de Sanidad, y, con ello, se inició un auge paulatino de la Sanidad interior ${ }^{40}$. No obstante, con anterioridad, en 1885, por R.D. de 20 de noviembre, se había creado el Cuerpo médico de Vacunadores del Estado ${ }^{41}$.

Además de las medidas legislativas referidas con las que se pretendió introducir mejoras en lo que a infraestructura y personal de vacunación se refiere, la I República dictaría otras con la finalidad de establecer un registro estadístico de los estados de vacunaciones, revacunaciones y de los casos de viruela que se produjeran en cada provincia ${ }^{42}$. Sin embargo, no siguió el ejemplo de Prusia, en donde el 2 de abril de 1874 se había aprobado la Ley de vacunación obligatoria, limitándose a disponer por Orden de 30 de diciembre de 1873 «la vacunación y revacunación de cuantas personas estén bajo la dependencia de las Autoridades», y excitando a éstas «a extender la vacunación allanando resistencias y destruyendo preocupaciones» ${ }^{43}$. A tenor de estas últimas palabras reproducidas cabe pensar en la importancia que, de cara a extender la vacunación antivariólica, poseía la eliminación de las preocupaciones existentes entre el vulgo y algunos médicos acerca de la ineficacia de la vacuna, y de la posible transmisión de otras enfermedades por medio de la vacuna. De ahí que no nos deba extrañar que aparecieran obras originales o traducciones ${ }^{44}$ y se desarrollaran debates científicos con el objetivo de desterrar dichas ideas y recelos frente a la vacunación antivariólica ${ }^{45}$, así como con

39 De hecho, no existió una cierta preocupación gubernamental por organizar «la Sanidad terrestre y marítima» y constituir un cuerpo de funcionarios de Sanidad Civil hasta 1879, si bien la propuesta no se materializó. Molero MeSA, J.; JimÉnEZ LuCEnA, I. (2000), p. 46.

40 Molero Mesa, J.; Jiménez LuCENA, I. (2000), p. 47.

41 Molero MesA, J.; Jiménez LucenA, I. (2000), p. 56. Como han señalado, J. Molero e I. Jiménez, dicho Cuerpo estaba integrado en ese momento por los 16 médicos del Instituto de Vacunación.

42 Dirección General de SANidAd (1903), pp. 79-81.

43 DireCCIÓN GENERAL de SANIDAD (1903), p. 78.

44 Una de estas traducciones fue: AVERVEK (1881).

45 Una muestra de ello, fueron: SiERra CABO, A. (1885), Apuntes de vacunología, Madrid, Impr. E. Teodoro; SERRET, R. (1887), Vacunología. Sobre la transmisibilidad de enfermedades por medio de la vacuna, Madrid, Administración de El Genio Médico-Quirúrgico. 
la intención de ir creando un estado de opinión favorable a la vacunación obligatoria. Sin embargo, ésta tampoco llegaría cuando, bajo la presión de la epidemia de viruela de 1890 , se dictó el R. D. de $1891^{46}$. En él, se indicaba con mayor precisión las edades de vacunación y revacunación, las medidas a adoptar en tiempo de epidemia, se recogían los cambios legislativos habidos en la vacunación antivariólica en los últimos años del siglo XIX, sobre todo tras la creación del Instituto de Vacunación, etc.. Además, conscientes de las dificultades para lograr su cumplimiento, se solicitaba la colaboración de las autoridades, los Centros libres de propaganda e Instrucción profesional, los Jefes de los servicios y organismos del Estado, así como de los Profesores Médicos. De hecho, la colaboración de estos últimos en la extensión de las vacunaciones y revacunaciones, así como mediante la creación de centros privados permanentes de vacunación, se premiaba con diferentes distinciones y preferencia para ascender y optar a plazas por concurso ${ }^{47}$.

De esta manera comenzaba una nueva etapa, marcada por el contenido del Decreto de 1891, del que hemos resaltado algunos aspectos, contando para su aplicación con un Instituto Nacional de Vacunación, un Cuerpo de Médicos Vacunadores del Estado y el libre mercado de la vacuna reconocido desde 1872. En las próximas páginas mostraremos hasta qué punto estas nuevas condiciones, que incluían también una campaña de propaganda favorable a la vacunación, se tradujeron en un mayor éxito en la lucha contra la viruela.

\section{CONTEXTO SANITARIO, GESTACIÓN Y DICTADO DEL R. D. DE 1903}

Con el Decreto de 1891 se inicia la última década decimonónica en la que, ante el conflicto de Cuba y su resultado final, cobrarán más fuerza algunos elementos que estaban operando desde, al menos, la década anterior. Me refiero sobre todo a la configuración del movimiento regeneracionista, en conexión con la mentalidad positivista imperante en nuestro país ${ }^{48}$ y muy in-

46 Como figura en la exposición del citado Real Decreto, esta disposición legal se dictaba mientras se preparaba la presentación de un Proyecto de ley. Exposición. «Real Decreto de 18 de agosto de 1891», Gaceta de Madrid, 234, 22 de agosto de 1891, 632-633, p. 632. Este R.D. sirvió en parte de modelo para confeccionar el articulado del R.D. de 1903.

47 «Real Decreto de 18 de agosto de 1891», Gaceta de Madrid, 234, 22-8-1891, 632-633, p. 633.

48 El positivismo desempeñó la importante función ideológica de legitimar el nuevo régimen. Abellán, J.L. (1989), Historia crítica del pensamiento español, vol. 5/1 [La crisis contemporánea (1875-1936)], Madrid, Espasa-Calpe, p. 74. 
fluido por la experiencia brindada por el sexenio revolucionario y por lo ocurrido en Francia tras la derrota de $1870^{49}$. El desarrollo paralelo, junto a la configuración del movimiento regeneracionista, de otras alternativas sociopolíticas como el socialismo, el anarquismo o el catalanismo, que se mostraron con solidez en la coyuntura de 1898, permitió realizar un proceso de revisión crítica y una reformulación de los problemas sociopolíticos que afectaban secularmente a España. De ahí el protagonismo que nuestra desatención sanitaria alcanzó y la influencia que ello tuvo en distintos aspectos como el de la lucha contra la viruela. A ello contribuyó igualmente el importante protagonismo que, en este movimiento regeneracionista, cobraron los profesionales sanitarios, que, como grupo social, habían alcanzado ya una cierta entidad y presencia pública ${ }^{50}$. Convencidos los médicos de que el desastre de $1898 \mathrm{y}$, en general, nuestro estado de crisis nacional se debía a nuestra mala situación sanitaria ${ }^{51}$, denunciaron dicha situación y plantearon sus propuestas reformis$\operatorname{tas}^{52}$ para lograr la modernización científico-sanitaria del país. En su opinión, la creación de nuevas instituciones científicas y una profunda reforma sanitaria, cuyo inicio debía ser la promulgación de una nueva Ley de Sanidad adaptada a los nuevos dictados de la ciencia médica ${ }^{53}$, eran las piezas claves para conseguir dicha modernización.

En un marco como el descrito, en el que, como se ha visto, se habían introducido algunas transformaciones en la lucha contra la viruela al hilo de las realizadas en lo que a nuestra organización sanitaria y situación de la clase médica se refiere, la viruela seguía siendo, sin embargo, un importante problema sanitario. De ahí que no nos deba extrañar que se denunciara su presencia $^{54}$ y nuestra elevada mortalidad por enfermedades infecciosas, superior

49 Acerca de la influencia que lo sucedido en Francia en 1870 tuvo en los intelectuales de nuestro país, resulta muy ilustrativo: CACHO VIU, V. (1997), Repensar el noventa y ocho, Madrid, Biblioteca Nueva.

50 Villacorta Baños, F. (1989), Profesionales y burócratas, Madrid, Siglo XXI, p. 76

51 Pulido Fernández, Á. (1899), Discursos parlamentarios sobre el Proyecto de Bases para una Ley de Sanidad, Madrid, Impr. E. Teodoro, pp. 28-29.

52 Estas propuestas se superpusieron, en muchos casos, sobre los viejos objetivos del reformismo profesional. VILLACORTA BAÑOS, F. (1989), p. 76. En este sentido, un ejemplo muy ilustrativo sería el trabajo de PULIDO FERNÁNDEZ, Á. (1902).

53 HAUSER, Ph. (1902), Madrid bajo el punto de vista médico-social, 2 vols., Madrid, Est. Tip. Sucesores de Rivadeneyra, vol. 2, pp. 7-11, (cito por la edición preparada por Carmen del Moral (1979), Madrid, Editora Nacional); PULIDO FERNÁNDEZ, Á. (1902).

54 RevengA, R. (1904), La muerte en España, Madrid, pp. 104-117. El problema de la elevada mortalidad por viruela en España en los primeros años del siglo XX fue denunciado 
a la de la mayoría de los países de Europa y América ${ }^{55}$. Esta vergonzosa presencia de la viruela se relacionaba con el atraso sanitario de nuestro país, la resistencia e indiferencia de la población hacia la vacunación, la falta de una legislación en la que se estableciera la obligatoriedad de la vacunación y revacunación antivariólica y, sobre todo, la falta de voluntad de las diferentes instancias de la Administración en hacer cumplir la legislación. De ahí que las actuaciones de los últimos años del siglo XIX y primeros del XX estuvieron dirigidas, por un lado, a la elaboración y presentación al Parlamento de Proposiciones de Ley al objeto de lograr la aprobación de una nueva Ley General de Sanidad ${ }^{56}$; y, por otro, a la demanda de nuevas instituciones científicas. Si el fracaso presidió los intentos de conseguir una nueva Ley de Sanidad, algunos logros se obtuvieron en lo que a instituciones científicas se refiere. En efecto, mediante el R.D. de 23 de octubre de 1894, el ministro de la Gobernación Alberto Aguilera (1840-1913) creó «un Instituto Nacional de Bacteriología e Higiene», que incluía entre sus funciones la vacunación preventiva contra la viruela ${ }^{57}$. Sin embargo, el ambiente de recelo presente en ciertos sectores de las profesiones sanitarias (médicos, farmacéuticos y veterinarios) que

en: Martín Salazar, M. (1913), La Sanidad en España, Madrid, Impr. Real Colegio de sordomudos y ciegos, pp. 137-138; y HAUSER, Ph. (1913), La Geografía médica de la Península Ibérica, Madrid, Impr. de Eduardo Arias, t. 2, pp. 260-267. Sobre la presencia de la viruela en Madrid, véase: HAUSER, Ph. (1902), vol. 2, pp. 45-53.

55 Oldamont (1901), La Higiene Pública en España, Madrid, A. de San Martín, p. 5; Pulido Fernández, Á. (1902); REvengA, R. (1904), pp. 7-20 (en ellas se recogen las cifras de mortalidad general desde 1858 hasta 1901); REAL CONSEJO DE SANIDAD (1901), Cuestiones fundamentales de Higiene Pública en España, Madrid, Est. Tip. de E. Teodoro. La elevada mortalidad en España había sido objeto de otros informes anteriores del mismo Consejo de Sanidad en 1884 y 1888 . Con posterioridad, la gran morbilidad y mortalidad por enfermedades infecciosas, superior a otras naciones de Europa y América, fueron igualmente denunciadas en los siguientes documentos legislativos: «Proyecto de Ley de Epidemias», El Siglo Médico, 3181, 28-11-1914, 762-764; «Real Decreto de 10 de enero de 1919 en relación con la prevención de las enfermedades contagiosas», Med. Soc. Esp., 4, (1919), 69-75; «Proposición de Ley del Sr. Van-Baumberghen, sobre profilaxis pública de las enfermedades infecciosas», Diario de Sesiones de Cortes. Congreso de los Diputados, Apéndice 5, 21, 30-7-1919, 1-13; «Proyecto de ley presentado por el señor ministro de la Gobernación, sobre profilaxis pública de las enfermedades infecciosas», El Siglo Médico, 3442, 29-11-1919, 1041-1045.

56 Algunas de estas propuestas fueron las de 1894-5, la de 1896 y la de 1899. «Proyecto de Ley de Bases», La Farmacia Española, 27, 5-7-1894, 421-425; «Real Orden del Ministerio de la Gobernación 816-3-1899)», La Farmacia Española, 12, 23-3-1899, 181-182; «Proyecto de Ley de Sanidad», La Farmacia Española, 32, 10-8-1899, pp. 497-503.

57 Gaceta de Madrid, 24-10-1894, p. 255. 
había rodeado su creación, impidió su materialización, siendo preciso esperar hasta 1899 para contar con el «Instituto de Sueroterapia, Vacunación y Bacteriología de Alfonso XIII», creado por Cortezo (1850-1933) ante la presencia de la peste en Oporto ${ }^{58}$. Con el nuevo Instituto, que venía a ocupar el hueco dejado por los disueltos Institutos Central de Bacteriología e Higiene y el de Vacunación del Estado, se buscaba modernizar la lucha contra las enfermedades infecciosas en nuestro país ${ }^{59}$.

Con el fin de vencer las resistencias e indiferencia frente a la vacunación antivariólica por parte de la población y de algunos médicos, continuó con más fuerza el debate sobre la viruela y la vacunación antivariólica. De hecho, este tema fue objeto de tesis doctorales ${ }^{60}$, memorias presentadas a las Academias de Medicina ${ }^{61}$ y diversas publicaciones - algunas claramente propagandísticas ${ }^{62}$ - aparecidas en torno a 1898, año éste del IX Congreso Internacional de Higiene y Demografía, celebrado en Madrid, y en el que la vacunación antivariólica y su obligatoriedad se constituyó en uno de los grandes protagonistas. Entre los españoles defensores de la vacunación obligatoria figuraron el Secretario de la Real Academia de Medicina Manuel Iglesias, directores de Institutos oficiales y centros privados de vacunación, médicos de la Beneficencia municipal y médicos titulares, siendo comúnmente aceptada la necesidad de solicitar de los Poderes públicos la aprobación de una ley de vacuna-

58 Ministerio de la Gobernación. Dirección General de SANidad (1900), Real Decreto de creación del Instituto de Sueroterapia, Vacunación y Bacteriología de Alfonso XIII y Reglamento para su aplicación, Madrid, Impr. Sucesora M. Minuesa de los Ríos, pp. 7-9.

59 Este tema ha sido abordado por mí en: PORRAS GALlO, Mª I. (1998), «Antecedentes y creación del Instituto de Sueroterapia, Vacunación y Bacteriología de Alfonso XIII», Dynamis, 18, 81-105.

60 Una de ellas fue la de Torre S. Somoza, C. de (1894), Profilaxis de la Viruela. Vacu$n a$, Santiago, Est. Tip. de José M. Paredes, en la que el autor terminaba suscribiendo la siguiente conclusión: «La vacuna constituye, con los medios higiénicos de aislamiento, el único tratamiento profiláctico de la viruela».

61 Una muestra de este tipo de obras fue la memoria, premiada por la Academia de Medicina, de CARBOnEll y SolÉs, F. (1898), Estudio comparativo, experimental y clínico de la viruela en el hombre y en los anímales domésticos, Barcelona, Tip. de la Casa Provincial de Caridad.

62 Un ejemplo lo constituye el folleto propagandístico de Gorgonio González Araco, director del Instituto de vacunación de la calle Valverde, uno de los centros privados existentes en Madrid desde 1886. Dicho folleto, en el que se defendía con entusiasmo la vacunación y revacunación hasta llegar a hacerla obligatoria, tuvo al menos dos ediciones: GONZÁLEZ ARACO, G. (1893), La vacunación antivariólica, Madrid, Tip. de Baena Hermanos; GonZÁlEz ARACO, G. (1898), La vacunación antivariólica, Madrid, Impr. de los Hijos de J.M. Ducazal. 
ción obligatoria en aquellos países que carecieran de ella ${ }^{63}$. Éste era el caso de España en el momento de celebrarse el citado Congreso, a pesar de haberse presentado al Congreso de los Diputados una Proposición de ley declarando obligatoria la vacunación y revacunación en $1895^{64} \mathrm{y}$ del creciente estado de opinión favorable al establecimiento de la obligatoriedad de la vacuna, desde la epidemia de viruela registrada en 1896. De hecho, esta epidemia motivó que el tema central de las sesiones de la Sociedad Española de Higiene fuera la vacunación forzosa, estudiándose un proyecto de Ley acerca del particular que se decidió someter a las Cámaras Parlamentarias ${ }^{65}$. Probablemente, ese Proyecto fue el presentado por el Secretario de la Real Academia de Medicina al Senado en $1897^{66}$, que, al igual que el citado anteriormente, tampoco sería aprobado $^{67}$. Con posterioridad no hubo más iniciativas similares, si bien Pulido, al ser nombrado Director General de Sanidad en 190168, anunció que se

63 Iglesias Díaz, M. (1900), Vacunación obligatoria. En: SAlcedo Ginestal, E. (dir.), Actas y Memorias del IX Congreso Internacional de Higiene y Demografia, celebrado en Madrid en los días 10 al 17 de abril de 1898, t. II (Higiene), Madrid, Impr. de Ricardo Rojas, pp. 91-95; ANTIGÜEDAD DíEZ, F. (1900), Vacunación obligatoria. En: SAlCEDo Ginestal (dir.), p. 96; BALAgUer (1900), Datos en pro de la vacunación y revacunación obligatorias. Epidemias de viruela sufridas en Madrid en los años de 1890 y 1896. En: SAlCEDo GINESTAL (dir.), pp. 99103; Ruiz, S. y Alarcón MANesCAU, J. de (1900), Notas del Instituto de Vacunación de Málaga. En: SAlCEDo GineSTAL (dir.), pp. 108-115; LlORENS, J. (1900), Vacunación. En: SAlCEdo Ginestal (dir.), pp. 121-124; Codina CASTellví, J. (1900), Necesidad de la revacunación repetida fundada en la ley etática de la viruela. En: SALCEDO GINESTAL (dir.), pp. 71-75; Dios SiMANCAS, J. de (1900), Vacunación obligatoria. En: SALCEDO GINESTAL (dir.), pp. 75-82.

64 «PROPOSICIÓN de Ley, del Sr. Ávila, declarando obligatoria la vacunación y revacunación», Diario de las Sesiones de Cortes. Congreso de los Diputados. Legislatura de 1894, Apéndice 11 al no 93, 1-4-1895, 1-4. La proposición se tomó en consideración y se nombró una Comisión que llegó a hacer un Dictamen, pero no prosperó, «DICTAMEN de la Comisión acerca de la proposición de ley declarando obligatoria la vacunación y revacunación», Diario de las Sesiones de Cortes. Congreso de los Diputados. Legislatura de 1894, Apéndice 21 al n 140, 8-6-1895, 1-4.

65 Belmás, M. (1897), Discursos leidos en la sesión inaugural del año académico de 1897-98 en la Sociedad Española de Higiene, Madrid, Escuela Tipográfica del Hospicio, 916 , p. 11.

66 «Proposición de ley del Sr. Iglesias y Díaz, estableciendo la vacunación y revacunación obligatoria», El Siglo Médico, 26-5-1897, 364-365.

67 CARlÁn, D. (1903), «Un Real Decreto importante», El Siglo Médico, 2563, 25-11903, p. 50.

${ }^{68}$ Información sobre la labor de Pulido en este período, figura en: GARCÍA GUERRA, D.; Álvarez AntuÑa, V. (1994), «Regeneracionismo y Salud Pública. El bienio de Ángel Pulido al frente de la Dirección General de Sanidad (1901-1902)», Dynamis, 14, 23-41. 
proponía redactar un decreto y, si eso no bastaba, un proyecto de ley de vacunación obligatoria ${ }^{69}$. Sin embargo, Pulido abandonaría dicho cargo sin llegar a realizar lo anunciado, y sería su sucesor, Carlos $\mathrm{M}^{\mathrm{a}}$ Cortezo, el responsable del R.D. de 15 de enero de $1903^{70}$. Éste, aunque fue publicitado como Real decreto sobre vacunación y revacunación obligatorias, como muy bien denunció Martín Salazar años más tarde en su discurso de ingreso a la Real Academia de Medicina, esto era así tan sólo en caso de epidemia o de recrudecimiento de la endemia ${ }^{71}$. Además, en opinión de este médico, la propia redacción del Decreto hacía que, incluso en esos casos, su aplicación ofreciera más dificultades por cuanto la norma legislativa descendía y se desvanecía «en múltiples detalles casuísticos que condicionan su cumplimiento y que complican más bien que facilitan su ejecución ${ }^{72}$. Probablemente, este hecho se debía a que, como señaló el propio Cortezo, ante la imposibilidad de aprobar ninguna de las dos proposiciones de ley de vacunación obligatoria que habían sido presentadas al Congreso y Senado, en el R.D. de 1903 se habían «limitado a reproducir en parte y a ampliar muchísimo» el R.D. de $1891^{73}$.

\section{APLICACIÓN DEL DECRETO DE VACUNACIÓN Y REVACUNACIÓN DE 1903}

Para proceder a la aplicación del Decreto de 1903 en las situaciones y condiciones indicadas se contaba en esos momentos con el ya mencionado Instituto de Alfonso XIII, algunos laboratorios provinciales y municipales - propios o subvencionados - y con un número progresivamente mayor de centros privados. Continuaba sin aprobarse una nueva Ley de Sanidad, pero se había dictado el R.D. de 31 de octubre de 1901 de Declaración Obligatoria de Enfermedades infecciosas y, aunque desde 1892 se contaba con los Inspectores Provinciales de Sanidad y la Sanidad interior había cobrado un cierto auge, seguían siendo muchas las carencias de nuestra Sanidad. De hecho, como ha señalado Rodríguez Ocaña, se estaba iniciando entonces la etapa que él ha denominado de «formación» de la Salud Pública española con la aprobación

69 CArlán, D. (1901), «Banquete al Sr. Pulido», El Siglo Médico, 2468, 14-4-1901, 225 226, p. 226.

70 «Ministerio de la Gobernación. Real Decreto», El Siglo Médico, 2563, 25-1-1903, 61-63.

71 MARTín SALAZAR, M. (1913), pp. 137-138.

72 MARTín SAlazAR, M. (1913), p. 138.

73 Cortezo hizo esas manifestaciones, bajo el seudónimo de Decio Carlán. CARLÁn, D. (1903), p. 49. 
de la Instrucción General de Sanidad al término de $1903^{74}$. Pues bien, en esos momentos, nuestro país se encontraba sumido en una profunda crisis política, económica y social.

En unas condiciones como las descritas y dados los fines de este trabajo, me parece interesante tratar de averiguar hasta qué punto fue viable la aplicación del nuevo decreto de vacunación y en qué medida ello pudo acompañarse de algún cambio en la mortalidad por viruela de nuestro país. En este sentido, además de los testimonios contemporáneos de médicos e higienistas y del examen de las tasas anuales de mortalidad por viruela correspondientes al primer tercio del pasado siglo, una vía apropiada para ello puede ser lo sucedido durante la epidemia de viruela de 1903-4, registrada en Madrid pocos meses después de dictarse el nuevo decreto de vacunación ${ }^{75}$. A tenor del testimonio del Vicepresidente de la Junta Provincial de Sanidad de Madrid, José Monmeneu, las medidas contenidas en dicho decreto se habrían aplicado inmediatamente. De hecho, al registrarse en agosto de 1903 un aumento de la mortalidad por viruela en Madrid, las autoridades ordenaron la vacunación y revacunación de todas las personas a quienes gubernativamente se podía imponer dicha medida profiláctica ${ }^{76}$. Sin embargo, parece que algo muy distinto fue tratar de lograr su cumplimiento. En opinión de Monmeneu, esto fue así «porque fácilmente se comprende que no es posible vacunar en breves días a una población de más de medio millón de habitantes ${ }^{77}$, ni siquiera a las 200.000 personas que al inicio de la epidemia se estimaba no habían sido sometidas a la profilaxis antivariólica ${ }^{78}$. En este sentido, además del papel que en ello pudo representar el enfrentamiento registrado entre partidarios y opositores de la vacunación durante una situación de epidemia, parece apropiado aceptar que la escasez de linfa fuera el principal impedimento para efectuar

74 Aunque este autor había situado dicho inicio en la creación del Instituto de Alfonso XIII en 1899, con posterioridad ha considerado más representativo de dicho inicio la aprobación de la Instrucción General de Sanidad al término de 1903, que marcará un cambio cualitativo en nuestra Sanidad, si bien el hecho de no ser ley limitó su operatividad. RODRíGUEZ OCAÑA, E. (2001), La Salud Pública en la España de la primera mitad del siglo XIX. En: AtENZA FernáNDEZ, J.; MARTínEZ PÉREZ, J. (coords.), El Centro secundario de Higiene rural de Talavera de la Reina y la Sanidad española de su tiempo, Toledo, JCCM, 21-42, pp. 25-29.

75 Información bastante completa e ilustrativa acerca de dicha epidemia, figura en: MONMENEU Y LÓPEZ REYNOSO (1904), La epidemia de viruela en 1903-4. Informe presentado a la Excma. Junta Provincial de Sanidad de Madrid, Madrid, Imprenta y Librería de Nicolás Moya.

76 MONMENEU Y LÓPEZ REYNOSO (1904), p. 15.

77 MONMENEU Y LÓPEZ REYNOSO (1904), p. 16.

78 MONMENEU y LÓPEZ REYNOSO (1904), p. 26. 
una vacunación masiva y, con ello, aplicar el decreto. De hecho, como refiere el citado médico, ni las existencias del Instituto de Alfonso XIII ${ }^{79}$, ni las aportaciones del Instituto de Higiene militar ni las de los dos Institutos madrileños privados - el de Balaguer, que prestaba el servicio a la Diputación y al Municipio; y el de González Araco - bastaron para atender a la demanda. De ahí que fuera preciso la importación de linfa de otros puntos de España y del extranjero ${ }^{80}$. Igualmente, buscando un mayor cumplimiento, se llevó por primera vez gratuitamente la vacunación a los domicilios privados ${ }^{81}$. A pesar de ello y de la buena voluntad mostrada por la población frente a la vacunación, se realizaron como máximo unas 65000 vacunaciones y revacunaciones durante la epidemia. Con ser muy importante esta cifra, sin embargo, suponía que al término de la epidemia en marzo de 1904, había aún como mínimo unos 135.000 individuos sin vacunar y, por tanto, susceptibles de padecer la viruela $^{82}$. Esta situación unida al cese de algunas medidas - como la vacunación a domicilio - en cuanto se vio descender la epidemia, propiciaban que la viruela siguiera siendo un problema sanitario en Madrid y pudiera provocar nuevas epidemias ${ }^{83}$. Como muestran los testimonios de Hauser y Martín Salazar en 1913, parece que esta situación no habría sido exclusiva de la capital de España sino bastante más general. De hecho, Hauser, sirviéndose de la tasa

79 En este sentido, cabe señalar las dificultades que este Instituto, dadas sus limitaciones presupuestarias, de infraestructura y de personal, tuvo para cumplir sus funciones en los primeros años de su existencia. Por otro lado, también es preciso recordar las campañas de desprestigio que, por intereses profesionales y mercantilistas, sufrió en ese período. PORRAS GALLO, MaI. (1998), p. 103.

80 Monmeneu y LóPEZ ReYNOSO (1904), pp. 17-18.

81 Esto fue señalado por Monmeneu. MonMENEU y LóPEZ REynoso (1904), p. 18. Sin embargo, según Chicote, parece que esto se habría realizado ya con motivo de la epidemia de 1900. CHICOTE, C. (1904), La lucha contra la viruela. Sobre la preparación de la vacuna en el laboratorio municipal de Madrid, Madrid, Impr. Municipal, p. 4. Información más completa sobre el Servicio Municipal de Vacunación a domicilio, figura en: CAMPOS MARín, R. (2001), pp. 13-15.

82 Monmeneu y López Reynoso (1904), p. 26.

83 De hecho, hasta que se decretó la vacunación antivariólica obligatoria en 1919, alcanzó carácter epidémico en Madrid en 1909, 1913 y 1918 (coincidiendo parcialmente con el segundo brote de la epidemia de gripe). LASBENNES, L. (1914), La viruela en Madrid, Madrid, Impr. Municipal; PORRAS GALlo, M ${ }^{\mathrm{a}}$ I. (1996), Evolución de la mortalidad de Madrid en el período 1883-1925: una aproximación a la realidad sanitaria que conoció Philiph Hauser. En: CARRILlO, J.L. (ed.), Entre Sevilla y Madrid: Estudios sobre Hauser y su entorno, Sevilla, A. Pinelo, 101129; PORRAS GALlo, Ma' I. (2002), «Un acercamiento a la situación higiénico-sanitaria de los distritos de Madrid en el tránsito del siglo XIX al XX», Asclepio, LIV-1, 219-250, p. 239. 
de mortalidad por viruela de las distintas provincias españolas durante el septenio 1900-1906, mostraba que era endémica en nuestro país y responsable de periódicas epidemias, sobre todo en las grandes capitales ${ }^{84}$. Por su parte, Martín Salazar, consignando las cifras de la mortalidad anual de España correspondientes a los dos primeros lustros del pasado siglo, aunque reconocía la mejoría que se había experimentado en el segundo con respecto al primero, creía que la intensidad y extensión de la viruela en España nos colocaba, expresado con sus propias palabras, «en una triste situación de inferioridad con relación a la mayor parte de los demás países de Europa y América» ${ }^{85}$.

Los testimonios que acabamos de referir se corroboran al examinar las tasas de mortalidad anual por viruela en España entre 1901 y 1930. Como se puede ver en la gráfica adjunta ${ }^{86}$, tras el Decreto de vacunación de 1903, la viruela siguió siendo un problema sanitario para nuestro país durante más de veinte años. De hecho, no se produjo su total desaparición hasta 1929, habiendo reaparecido tan sólo al final de la Guerra Civil y en los años de nuestra inmediata posguerra (1939-1942) ${ }^{87}$. Ahora bien, desde 1903 se observa una tendencia descendente de la mortalidad por viruela, interrumpida tan sólo por la presentación de algunas epidemias, y que se acentuó tras la epidemia registrada en torno a los años de la pandemia de gripe de 1918-1988.

A la vista de la evolución de la mortalidad por viruela en el primer tercio del siglo XX, cabe preguntarse acerca de las razones que, por un lado, justificaban su presencia cuando en la mayoría de los países europeos había sido eliminada; y, por otro, su posterior descenso y desaparición. En opinión de Hauser, la indiferencia de los habitantes frente a los medios preventivos contra la viruela era uno de los elementos que explicaba la persistencia de esta enfermedad en nuestro país. Sin embargo, con respecto a esto, conviene recordar, por un lado, que Monmeneu había remarcado la buena disposición frente a la vacunación e incluso el estado de docilidad de los madrileños duran-

84 HAUSER, Ph. (1913), t. 2, pp. 260-267.

85 MARTín SALAZAR, M. (1913), pp. 137 y 135-136.

86 Elaborada por mí, a partir de los datos proporcionados amablemente por Ferrán Martínez Navarro, Jefe de Área de Vigilancia de Salud Pública de dicho centro (ISCIII)

87 Según los datos facilitados por Ferrán Martínez, las cifras de las tasas de mortalidad por viruela (por cien mil habitantes) durante esos años, habrían sido las siguientes: 2,4 (1939); 3,8 (1940); 0,7 (1941) y 0,1 (1942).

88 Esto mismo se advierte al examinar los datos recogidos por Pascua, aunque figuraran sólo cifras absolutas en vez de tasas. PASCUA, M. (1934), Mortalidad en España por rúbricas de la lista internacional abreviada de causas de defunción, y algunos otros indices de movimiento de población, Madrid, Dirección General de Sanidad, p. 8 y tablas. 


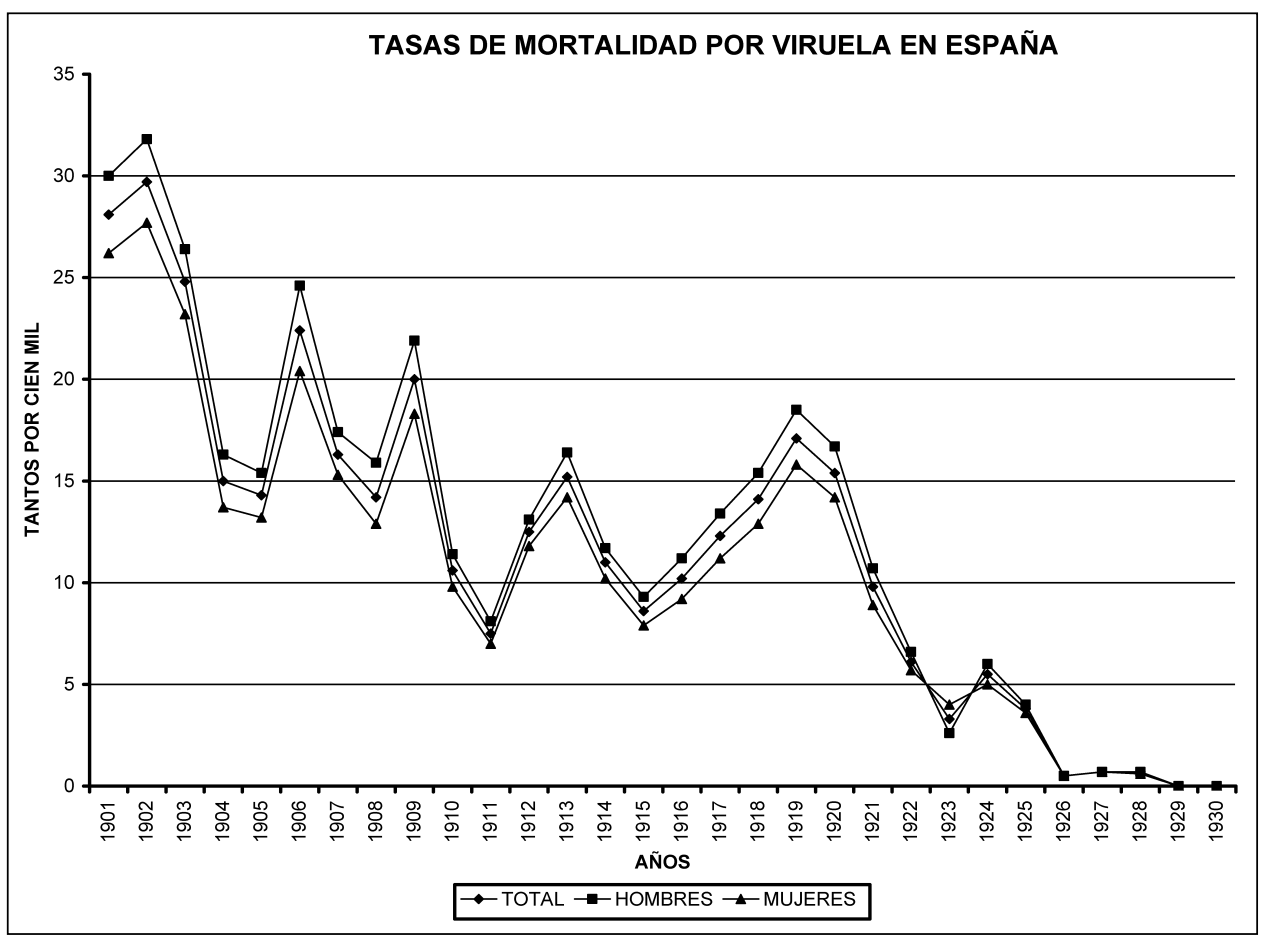

te la epidemia de $1903-489$; y, por otro, que Balaguer relacionó la resistencia de los padres a vacunar a sus hijos antes de los tres o cuatro meses de edad, con las opiniones mantenidas por algunos médicos a este respecto ${ }^{90}$. Junto a la indiferencia de la población, Hauser señaló la incapacidad de las autoridades para imponer la vacunación en las escuelas, a los empleados estatales, a los criados, etc., así como para hacer cumplir el Decreto de declaración obligatoria de enfermedades infecciosas ${ }^{91}$. Además de lo mencionado, el citado médico relacionaba la vergonzosa presencia de la viruela, sobre todo, con nuestras grandes carencias sanitarias, tanto en infraestructura (pabellones de aislamiento, hospitales de enfermedades infecciosas...) como en lo que a or-

89 Monmeneu y LóPez Reynoso (1904), p. 27.

90 Balaguer y Mayo, A. (1908), Vacunación de los recién nacidos, Madrid, Impr. J. Corrales, p. 3.

91 HAuser, Ph. (1913), t. 2, pp. 264-265. 
ganización y legislación se refería ${ }^{92}$. De hecho, Hauser consideraba que lo que realmente hacía falta en España era una nueva Ley de Sanidad adaptada a los conceptos modernos de la Higiene Pública ${ }^{93}$. Sin ella, entendía que todas las reformas sanitarias que se realizaran en nuestro país tendrían una eficacia muy limitada. Por su parte, Martín Salazar, que compartía también la necesidad de una nueva Ley de Sanidad, relacionaba la elevada mortalidad por viruela con las características del R.D. de 1903, señaladas en las páginas precedentes, especialmente con el hecho de que se renunciara a la vacunación obligatoria ${ }^{94}$. En su opinión, la rápida disminución de la mortalidad por viruela en todos los países donde había sido aplicada con rigor esta medida, justificaba el establecimiento de la obligación legal de la práctica vacunal en nuestro país ${ }^{95}$. Ahora bien, Martín Salazar juzgaba de enorme interés que, tras la aprobación de dicha norma legislativa, ésta realmente se cumpliera y, para ello, creía indispensable que tanto el Estado como las provincias y los municipios ofrecieran «gratuitamente vacuna buena y abundante a todo el mundo» ${ }^{96}$. De ahí que, además del Instituto Nacional de Higiene de Alfonso XIII, entendía que era preciso que todas las provincias y los grandes municipios contaran con sus propios Institutos de vacunación para atender y responder a sus propias exigencias ${ }^{97}$.

A tenor de los testimonios de Hauser y Martín Salazar, parece que la persistencia de la viruela en nuestro país se relacionaba, por un lado, con el hecho de que no se hubiera establecido la vacunación obligatoria y, por otro, con las importantes carencias de nuestra Sanidad que, como ya se ha visto, estaban siendo denunciadas por los médicos desde los inicios del pasado siglo. Pues bien, tras el fallido intento en 1911 de aprobación de una Ley General de Sanidad ${ }^{98}$, se ensayó una nueva vía para tratar de mejorar la situación sanitaria de nuestro país e iniciar la reforma de nuestra Sanidad: la de las leyes parciales o especiales ${ }^{99}$. A tal fin, se eligió la lucha contra las enfermeda-

92 HAuser, Ph. (1913), t. 2, p. 265.

93 HAUSER, Ph. (1913), t. 2, p. 267.

94 MARTín SALAZAR, M. (1913), pp. 137-139.

95 MARTín SAlazAR, M. (1913), p. 139.

96 MARTÍN SALAZAR, M. (1913), p. 144.

97 MARTín SAlazAR, M. (1913), pp. 143-144.

98 Pulido, Á. (1914), «La ley sobre epidemias y la asamblea de titulares», El Siglo Médico, 3180, 21 de noviembre de 1914, 739-740, p. 739; MARTín SALAZAR, M. (1913), pp. 71-85.

99 Esta vía de las leyes especiales o parciales se había utilizado en Alemania (1900), Prusia (1905), Gran Bretaña y Estados Unidos. PUlido, Á. (1914), p. 739; MARTín SAlAzAR, M. (1913), pp. 71-85; LEWIS, J. (1993), Public health doctors and AIDS as a public health issue. En: BerRidge, V. \& Strong, Ph. (eds.), AIDS and contemporary history, Cambridge, Cambridge University Press, 37-45, pp. 43-45. 
des infecciosas ${ }^{100}$, elaborando Martín Salazar el Proyecto de Ley de epidemias (1914), primer documento de estas características que recogía tanto la vacunación antivariólica obligatoria como el resto de las demandas realizadas por el autor del proyecto y por Hauser para lograr eliminar la viruela de nuestro país, así como otras relativas a la mejora de la situación de la profesión médica ${ }^{101}$. El éxito no acompañaría a esta primera iniciativa presentada a las Cortes, impidiendo la oposición ejercida por algunos de los diputados médicos y los partidos regionalistas su discusión y aprobación ${ }^{102}$.

Renovada actualidad cobrarían tanto la mala situación de la Sanidad española y la necesidad de reformarla como la profilaxis pública de las enfermedades infecciosas durante el desarrollo de la pandemia de gripe de 1918-19, que además coincidió parcialmente con una nueva epidemia de viruela (véase la gráfica adjunta). De hecho, este nuevo episodio varioloso reveló una vez más las dificultades que existían para aplicar el R.D. de 1903. A su vez, la gravedad alcanzada por la gripe motivó un importante debate sobre nuestras carencias sanitarias y los modos de resolverlas, que tuvo lugar en distintos escenarios, siendo uno de ellos el Parlamento. Allí, durante el desarrollo del segundo brote epidémico, se expresó la necesidad de promulgar una nueva Ley General de Sanidad, o bien la aprobación de una ley de profilaxis contra las enfermedades infecciosas ${ }^{103}$. De hecho, se elaboraron y presentaron al Congreso dos proyectos de Ley sobre profilaxis pública de las enfermedades infecciosas en julio y noviembre de $1919^{104}$. Ambos intentos resultaron fallidos, siendo sustituidos por el R.D. de 10 de enero de 1919 relativo a la prevención de las enfermedades infecciosas ${ }^{105}$, por el que se estableció la obligatoriedad de vacunarse contra la viruela «antes de los seis meses de edad», y

100 De este tema me he ocupado en: Porras Gallo, Ma I. (1994), «La lucha contra las enfermedades «evitables» en España y la pandemia de gripe de 1918-19», Dynamis, 14, 159-183.

101 «Ley de Epidemias», El Siglo Médico, [3181, 28-11-1914, 762-764; 3182, 5-12-1914, 777-780].

102 Aunque se contaba aparentemente con el apoyo de los médicos titulares, de los Subdelegados de Medicina y del partido liberal, los partidos regionalistas y otros sectores médicos no estaban conformes con algunos aspectos del proyecto. Más información, figura en: Porras Gallo, MaI. (1994), p. 169.

103 Porras Gallo, M M I. (1997), Un reto para la sociedad madrileña: la epidemia de gripe de 1918-19, Madrid, Ed. Complutense-CAM, pp. 87-88. Una información más completa sobre este tema, puede obtenerse en: Porras GALLO, Ma' (1996b), Una ciudad en crisis: la epidemia de gripe de 1918-19 en Madrid, Barcelona, ETD micropublicaciones, pp. 542-552.

104 Porras Gallo, Ma' I. (1994), p. 172.

105 Gaceta de Madrid, 23, 23 de enero de 1919, pp. 308-310. 
de revacunarse «cada siete años hasta los treinta», siendo igualmente obligatorio que las personas de más edad que no hubieran cumplido estos requisitos se sometieran a dichas prácticas ${ }^{106}$. De ahí que se pueda pensar que esa reducción de la mortalidad por viruela tras la epidemia de 1918-19 y su posterior desaparición podrían estar relacionadas con la correcta aplicación de las medidas contenidas en el citado decreto, $\mathrm{y}$, por tanto, habría que admitir el papel representado por la pandemia de gripe en la eliminación de la viruela. Por un lado, cabría señalar su papel como dinamizadora de una serie de medidas encaminadas a lograr mejoras socio-sanitarias ${ }^{107} \mathrm{y}$, por otro, como creadora de actitudes favorables a la vacunación contra la viruela, tanto entre la población como entre las autoridades. En este sentido, es interesante recordar la importante labor propagandística desarrollada por El Socialista, apoyando las campañas de vacunación antivariólica, especialmente al término del segundo brote de la pandemia ${ }^{108}$.

Ahora bien, junto a esta innegable contribución de la gran pandemia gripal de 1918-19 a la desaparición de la viruela, no se puede olvidar tampoco el papel representado por las transformaciones y mejoras que se fueron llevando a cabo durante la etapa de formación de la Salud Pública en España ${ }^{109}$, ni la repercusión que en todo ello tuvo la mejora económica experimentada en los primeros veinticinco años del pasado siglo en relación con el lento desarrollo de la Revolución Industrial habido en nuestro país.

106 Real Decreto de 10 de enero de 1919 en relación con la prevención de las enfermedades contagiosas, Medicina Social Española, 4, 69-75, p. 73.

107 Sobre las medidas adoptadas durante la epidemia de gripe de 1918-19 o inmediatamente posterior, véase: Porras Gallo, Mã. (1997), pp. 96-99; PORRAS Gallo, Ma I. (1996b), pp. 553-591.

108 El Socialista, 3421, 6 de diciembre de 1918, p. 3. El comportamiento de este diario obrero durante la pandemia de gripe de 1918-19 ha sido abordado en: PORRAS GALLO, M ${ }^{\text {a }}$ I. (1992), «La epidemia de gripe de 1918-19 en la prensa obrera». En: HUERTAS, R. y CAMPOS, R. (coords.), Medicina Social y clase obrera en España (Siglos XIX y XX), Madrid, FIM, 2 vols., vol. 1, pp. 125-144.

109 Rodríguez OCAÑA, R. (2001), pp. 25-29. Sobre este tema, puede verse también: RoDRíGueZ OCAÑA, R. (1994), «La Salud Pública en España en el contexto europeo, 18901925», Rev. San. Hig. Pub., 68, 11-27, pp. 20-25; Huertas GarcíA-Alejo, R. (1995), Organización sanitaria y crisis social en España, Madrid, FIM, pp. 27-59. 


\section{CONCLUSIONES}

A lo largo de las páginas precedentes, se ha mostrado cómo los factores profesionales, sanitarios, legislativos, políticos, económicos y sociales estuvieron relacionados con las dificultades habidas para generalizar la práctica vacunal en nuestro país a lo largo del siglo XIX e incluso de parte del XX, y cómo se fueron salvando los principales obstáculos poco a poco al hilo de las transformaciones que se iban realizando tanto en las circunstancias históricopolítico-sociales como dentro de la profesión médica y de la situación de nuestra Sanidad. Como se ha puesto de relieve, en ello tuvieron un destacado papel las epidemias (de viruela y otras enfermedades) registradas. De ahí que la desaparición de la viruela haya que relacionarla tanto con el efecto de la pandemia de gripe de 1918-19 como con las transformaciones realizadas durante la denominada etapa de «formación» de la Salud Pública española. 